\title{
An attitude-based reasoning strategy to enhance interaction with augmented objects
}

\author{
Josué Iglesias, David Gómez, Ana M. Bernardos, José R. Casar \\ Universidad Politécnica de Madrid, Telecommunications School \\ Madrid, Spain \\ \{josue, david.gomez, abernardos, jramon\}@grpss.ssr.upm.es
}

\begin{abstract}
This paper describes a mobile-based system to interact with objects in smart spaces, where the offer of resources may be extensive. The underlying idea is to use the augmentation capabilities of the mobile device to enable it as user-object mediator. In particular, the paper details how to build an attitude-based reasoning strategy that facilitates userobject interaction and resource filtering. The strategy prioritizes the available resources depending on the spatial history of the user, his real-time location and orientation and, finally, his active touch and focus interactions with the virtual overlay. The proposed reasoning method has been partially validated through a prototype that handles $2 \mathrm{D}$ and $3 \mathrm{D}$ visualization interfaces. This framework makes possible to develop in practice the IoT paradigm, augmenting the objects without physically modifying them.
\end{abstract}

Keywords-Internet of Things; augmented reality; contextual reasoning; location-based services; human-computer interaction

\section{INTRODUCTION}

Coined in 1999 by Kevin Ashton [1], the term Internet of Things (IoT) represents an attempt to extend the paradigm of Internet to the real world. While traditional Internet enables interaction with virtual resources, IoT aims at facilitating interaction with physical resources through virtual means. This paradigm opens the way to an automatic and intelligent exploitation of the information generated (i) by the objects themselves and (ii) by the user-object interaction history in order to provide services which may include physical feedback. IoT brings new capabilities, but also new challenges to overcome [2]; object information representation, user-object interaction and technologies to easily make objects smart are some of the most attractive for the research community.

Considering the interaction aspect, it seems obvious that one of the most direct ways for receiving information about a physical resource is by directly interacting with it; but most daily-life objects do not offer information representation capabilities. Nevertheless, users of smartphones and tablets are ramping up and the latest generations of mobile personal devices (with wireless communication capabilities, large screens and tactile interfaces) may enable new interaction paradigms with smart spaces. In particular, mobile-based augmentation systems, conceived to superimpose one or more digital overlays on reality, may be designed to facilitate browsing and interaction with objects. Digital overlays may be natively virtual, or compiled from real data coming from real objects.

Augmentation-based strategies for object representation and interaction can be applied to a variety of domains. In home automation, mobile augmentation may enable the delivery of energy consumption-aware services or make easier home appliance control [3]. It may also be used to simplify the learning and usage of unknown systems, facilitating operations and maintenance procedures. New marketing experiences may use spatially augmented services to offer updated information about a set of items (in a shop or even from the outside). A novel perspective to gaming and entertainment applications is possible through augmented strategies. A deep analysis of these and other use cases can be found in [4].

The delivery of a satisfactory user experience with usercentric ubiquitous augmented services presents some technological issues to be solved [2]. In particular, it is possible that, in a near future, the digital overlay on the real world to be greatly populated by millions of digital information pieces, being resource filtering and presentation technologies extremely necessary for augmented systems. Additionally, indoor deployments of augmented systems require accurate positioning technologies, precise representation (the user is very sensitive to errors) and an interaction model that considers the different expectations the user may have in terms of visualization.

Thus this paper presents an attitude-driven framework to facilitate interaction with augmented objects, both indoors and outdoors. The system is able to perform an intelligent selection of resources, being this process driven by the user's attitude, understood here as location, user-object proximity, relative orientation, resource visibility and interaction. The framework has been conceived from a generic perspective, providing $2 \mathrm{D}$ and $3 \mathrm{D}$ visualization tools. It aims at being easily extendable to different application domains and capable of dealing with different localization accuracies and resource visualization strategies.

Existing approaches for IoT-based resource representation and interaction are gathered in Section II. Section III introduces the main challenges for smart space augmentation and presents the design of our attitudereasoning system, whose architecture is detailed in Section IV. The prioritization method used for resource filtering is presented in Section V. The paper concludes with a review of the status of this development and future works and conclusions (Sections VI and VII).

\section{STATE OF THE ART}

Since the launch of the first commercial mobile browsers in 2009 (e.g., Layar ${ }^{1}$ or Wikitude ${ }^{2}$ ), augmentation systems

\footnotetext{
${ }^{1}$ http://www.layar.com
} 
for reality have become popular in smartphones. Following there is a review on how this technology has been explored in literature as a mean to interact with objects.

Augmented Reality (AR) and IoT has been considered for different purposes. For example, [2] contains a comprehensive analysis of IoT browsing necessities and proposes a typical augmented reality interface to monitor the status of a plant in a 'smart pot'. [5] contains a survey on how IoT may help people with disabilities. In particular, it describes a specific system called LinguaBytes [6] that stimulates children's language and communication skills with audio-video augmented everyday objects, so mentally handicapped children could consolidate, e.g., object-sound associations.

Augmented systems rely either on mobile sensors (position and inertial sensors) or on visual markers to detect the object's situation. Vision-based techniques, based on image analysis and pattern recognition, are usually slower and too heavy to be directly handled in the device. When applied to IoT, these systems should handle a broad database of objects to automatically recognize real instances [7]. Sensor-based strategies rely on GPS and inertial mobile technology when available. This approach automates service triggering without user collaboration, but it does not work properly when indoors (GPS signal is not available and inertial technology are affected by, e.g., metallic furniture). Additionally, users are much more sensitive to location errors when inside a building (references are visually close). Some other proposals use tags to easily detect the exact position of the user and augment objects. First approaches were based on bidimensional tag codes recognition (e.g., [8]), but they tend to be replaced by NFC tags due to its reusability and extra information storage capacity. For instance, Trinetra is a system for helping blind people (equipped with a RFID reader) to identify RFID-tagged items in a shop; once the item is recognized, the information is transferred to the smart phone, which turns the response into speech [9]. The 'smart pot' research previously mentioned [2] considers both QR-codes and RFID tags.

As previously stated, augmented resource representation services deployed in densely populated smart spaces may require a selection mechanism to filter the complete set of available resources in order not to burden the user with unnecessary information. This selection should be intelligent enough to be able to adapt the selection to each user particularities and circumstances, i.e., it should be contextaware. With respect to this issue, [10] offers a context-aware service recommendation system based on users' collective behaviour (specifically on collective positioning information). This work is further extended in [11], where historical records of user-device interaction are analysed in order to enhance the recommendation; a genetic algorithm is used in order to adapt the recommendation process to the user's circumstances. Equipped with special gadgets, the researches of [7] are able to determine where the user is looking at, exploiting this 'gaze tracking system' for enhancing the recommendation process. Of course, user's

\footnotetext{
${ }^{2}$ http://www.wikitude.com
}

(present and past) location can be also used as an input factor for recommendation [2][5][7].

How the icons (and information) associated to the resources to show are presented to the user is critical to attract user's attention and facilitate system interaction. Several projects propose to (de)emphasize the overlapped images by changing their characteristics (colour, shape, size, etc.) in order to adapt them to the user context [2][12][13]. Of course, not only images can be adapted, but also text [7] or even sounds [5].

The proposed work addresses both resource filtering and representation based on a context-aware prioritization method. Although the framework also considers static user and resource information processing, this paper focuses on exploiting user-resources relative attitude (i.e., resources location, user-resources proximity, relative orientation, resource visibility and interaction).

\section{Motivation: CHALlENGES IN RESOURCE VISUALIZATION IN DENSELY POPULATED SPACES}

From the previous literature review, and based on our own experience, we have compiled the following design considerations to develop our representation and interaction techniques for IoT-based smart spaces:

1) Automatic resource recognition and identification. Resources of interest (e.g., objects, spaces) should be automatically recognised and univocally identified in order to be able to correctly represent the resources in the user's device and also to record user-resource interactions.

2) Context-aware resource filtering. AR-based interfaces may result confusing if there are too many resources available, hindering user's experience if no filtering policy is established. This policy should be contextaware, i.e., adapted to users' and environments' particularities and circumstances.

3) Context-aware content building. The icons associated to the resources and the information to be shown may vary depending (i) on the characteristic of the resource to be augmented, (ii) on the consumer (user) particular circumstances or (iii) on external factors (e.g., time, weather, etc.). Then, the augmentation interfaces employed should be able to dynamically change in order to support this adaptation. Moreover, it could be even possible to dynamically decide what kind of representation paradigm to use at each moment depending on user's necessities and/or available technologies.

4) Spatial awareness. Knowing the relative position and orientation of users with respect to the surrounding resources is critical for augmentation systems. In general, indoor sensor-based augmented systems require a centimetre error, which is otherwise difficult to get from indoor positioning systems relying on simple radiofrequency systems (not needing the deployment of special extra infrastructure). It is usual to require the collaboration of the user to trigger an AR indoor service, 
either by asking him to proactively read a beacon or to mark his exact position. Orientation biases may also be more significant when indoors, due to the materials around, which have a distorting effect on the measurements. Thus, the visualization strategy has to take into account the quality of spatial inputs to provide suitable interfaces and feedback.

5) Map matching management. Augmented IoT services should be aware of the spatial particularities of the scenario where they are deployed. For instance, inter-zone relations ('zoneA adjoins zoneB' or 'zoneA can be accessed from $z o n e B$ ') are required to manage user line-of-sight (to determine which resources are visible and those that are not). Resource filtering processes may need this spatial information in order to prioritize resourees depending on their locations. Resource representation may also alter its behaviour depending on the physical visibility of a given resource.

6) User-resource interaction paradigm. Traditional mobile augmented reality requires a user to tap on a resource to activate its contents (explicit interaction). When handling visualization devices such as tablets, this interaction mechanism may be enhanced by, e.g., managing the time of focusing towards a given resource (implicit interaction). It is then important to define an interaction scheme for the augmentation system, depending on the type of services and visualization devices.

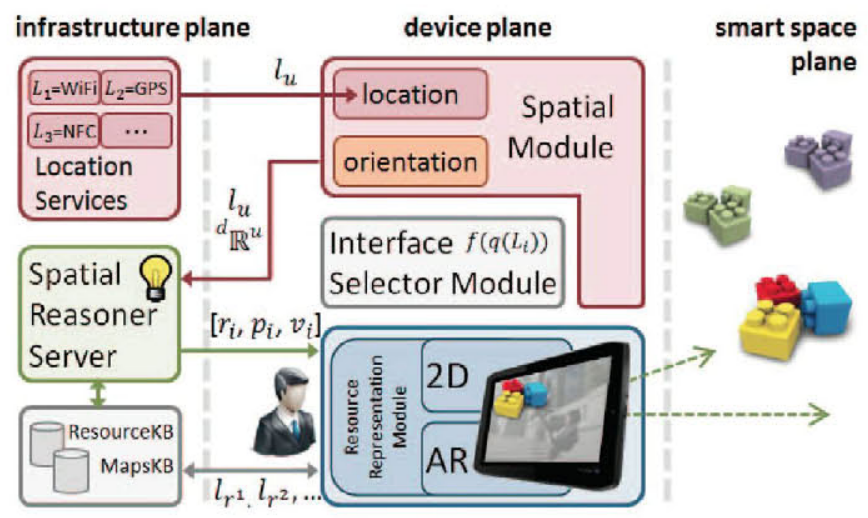

Figure 1. Framework architecture.

\section{ARCHITECTURE DESIGN}

All the aspects previously enumerated have been considered for designing the architecture of our particular augmentation framework.

Resources recognition and identification are based on their known position. Two different types of location estimation systems are available in order to be used to determine mobile resources position (e.g., users): (i) a fingerprint method based on WiFi signal strength measurements, providing coordinates with an approximate accuracy of 2-3 m. [14] and (ii) a beacon-based proximity system (RFID tags and pressure mats used to obtain accurate positioning -centimetre errors). Initially, we will assume that the resources to recognize or represent are static, not able to change their position continuously; this approach is reasonable for most application scenarios.

Two kinds of interfaces are considered: (i) an AR-based one would be offered if the precise location is available and (ii) a bidimensional one ('compass-based'), otherwise.

Our system considers explicit and implicit user-object interactions. Users can explicitly obtain information about an augmented object by touching its associated icon in any of the available interfaces. User-objects relative attitude is explode as implicit interaction; two factors are used in this sense: (i) user-object distance and (ii) user-object relative orientation.

Currently, the system only considers disjoint square zones coinciding with real zones (a hotel room, a meeting room, etc.). This is a first approach to be able to distinguish between the set of resources in the view of the user and those not visible (e.g., being behind a wall).

Both, the explicit/implicit interactions and the disjoint square zones map, feed a context-aware resource filter. It prioritizes (i) those resources in the view of the user, (ii) the resources closer to the user and (iii) those resources the user tend to 'focus' more with his/her mobile device.

\section{A. Execution planes}

The proposed system is composed by three planes (see Fig. 1):

- The smart space plane includes the set of all available resources. This considers (i) smart objects capable of offering information, (ii) those capable of receive information and (iii) passive resources. Every kind of resource may be potentially augmented.

- The device plane (embedded in the user's personal device) hosts all the processes required to (i) select the most suitable interface considering spatial estimators' quality, (ii) represent resources taking into account the line-of-sight from the user to the resource itself, (iii) visualize context-aware information related to the augmented resources and (iv) acquire spatial information, in particular to infer user-resource attitude.

- The infrastructure plane supports device plane execution offering (i) external location estimation systems, (ii) reasoning services for context-aware resources and information filtering and (iii) resources and maps knowledgebases.

\section{B. Information workflow}

Following Fig. 1, information workflow starts at the device plane when selecting the best available localization method $L_{i}$ to calculate $l_{u}$, the user's location. Location estimation is handled by a location fusion system that facilitates seamless handovers between positioning technologies, depending on their quality and availability [14]. As different interfaces require different location qualities, an Interface Selector Module is in charge of enable/disable the available interfaces depending on the selected location method quality $q\left(L_{i}\right)$. The AR-based interface would be only available if a location system with 
enough accuracy is active, on the other hand, location systems with lower quality may be used to support the $2 \mathrm{D}$ 'compass-based' representation (see Section VI for more details on representation issues). Device-embedded sensors (in particular, magnetometer and accelerometer) are used to obtain also ${ }^{d} \mathbb{R}^{u}$, the dynamic rotation matrix used to transform from the device to the user coordinates system (see Fig. 2). $l_{u}$ and ${ }^{d} \mathbb{R}^{u}$ are then sent to the Spatial Reasoner Server in the infrastructure plane.

The Spatial Reasoner Server is in charge of filtering the complete set of available resources of the smart space in order to select those more adapted to the user's circumstances. This selection is done using an incremental prioritization method (further described in Section V) exploding user's $\left(l_{u}\right)$ and resources' $\left(l_{r^{i}}\right)$ locations (stored in the Resources $K B$ ) and the spatial distribution of the place surrounding the user (MapsKB). This process generates a list identifying a subset of resources together with an associated priority value and a flag indicating if they are visible or not to the user $\left(\left[r^{i}, p^{i}, v^{i}\right]\right)$. This list is then sent back to the device plane.

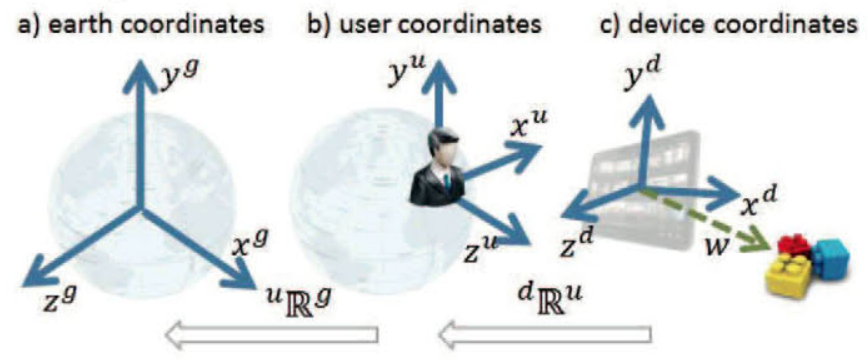

Figure 2. Involved coordinate systems.

Then, only those resources appearing in the priority list are shown to the user. The user's device is in charge of representing the icons associated to these resources by using the information about the user's attitude (i.e., $l_{u}$ and ${ }^{d} \mathbb{R}^{u}$ ) and the resources' position ( $\left.l_{r^{i}}\right)$, acquired from the Resources $K B$. Both, the priority value $p^{i}$ and the visibility flag $v^{i}$ are used by a context-aware Resource Representation Module to determine icons' characteristics used to augment the resources. Initially, resources that are not in the user's line-of-sight are represented in grey, but other aspects may be considered to vary the objects (e.g., colour, shape or size).

It has to be noted that device plane's resources are georeferenced using a global coordinates system with the Earth's centre as origin (Fig. 2.a). All the calculations, both for reasoning and for representation purposes, are performed using this global coordinates system and require ${ }^{u} \mathbb{R}^{g}$, the rotation matrix for transforming from the user to the global coordinate system. Being the user's position known, the calculation of ${ }^{u} \mathbb{R}^{g}$ is straightforward.

\section{ATTITUDE-BASED REASONING STRATEGY}

From the complete set of available resources only a selected subset will be represented in the user's device. This selection is based on a four-stage prioritization method. The first prioritization stage $p_{o}\left(u^{i}, r^{j}\right)$ is based on a recommendation method based on profiling such an itembased or collaborative recommendation system. This method is considered to be already available and remains out of the scope of this paper. Thus the output of this first stage will be a list of resources, ordered by priority taking into account the user's profile.

\section{A. History-based prioritization}

User's spatio-temporal records may enable history-based resource prioritization. Basically, this stage intends to introduce past data about the user's whereabouts and habits to adapt the resource offering. The history record is currently stored in a behaviour matrix $\mathbb{B}_{S \times T}$ representing the actual spatio-temporal probabilistic distribution of the user; whereas $\mathbb{B}$ rows represent discrete periods of time (e.g., hours), $\mathbb{B}$ columns represent available locations. This matrix offers probabilistic information regarding where and when the user tent to be.
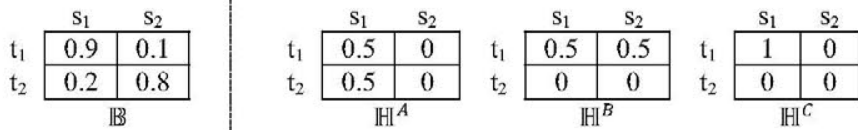

Figure 3. $\mathbb{B}$ and $\mathbb{H}$ matrices examples.

A new matrix $\mathbb{H}_{\mathrm{S} \times \mathrm{T}}$ is used to associate each available resource to the spatio-temporal distribution of the user. $\mathbb{H}$ has the same structure as $\mathbb{B}$ but it defines, however, a priority spatio-temporal distribution based on users' behaviours, i.e., what kind of user each resource is more adapted to (taking into account where and when the user tent to be). This configuration is flexible enough to allow resource prioritization by (i) user location (e.g., Fig. 3.A), (ii) time (Fig. 3.B) or (iii) spatio-temporal combinations (Fig. 3.C). Note that, for normalization purposes, $\sum_{\forall s, t} \mathbb{H}_{s t}=1$.

Although more complex metrics could be used in future extensions, history-based prioritization $p_{h}\left(u^{i}, r^{j}\right)$ is currently performed as a weighted sum (1) involving the $\mathbb{B}$ matrix of a particular user $u^{i}$ and the $\mathbb{H}$ matrix of a specific resource $r^{j}$. This equation also takes into account current time $\left(t^{c}\right)$ and user's current location $\left(s^{c}\right)$; the importance of historic versus current user's behaviour can be then balanced adjusting $h\left(r^{j}\right)$ and $a\left(r^{j}\right)$ weights (with $h\left(r^{j}\right)+a\left(r^{j}\right)=$ 1).

$$
p_{h}\left(u^{i}, r^{j}\right)=h\left(r^{j}\right)\left[\sum_{\forall s, t} \mathbb{B}_{s t}^{u^{i}} \cdot \mathbb{H}_{s t}^{r^{j}}\right]+a\left(r^{j}\right)\left[\mathbb{H}_{s^{c} t^{c}}^{r^{j}}\right]
$$

\section{B. Spatial-based prioritization}

User-objects relative attitude is also used to update the resources' priority. Two factors are considered in this stage: the user-resource distance $d_{u^{i}-r^{j}}$ and the user-resource relative angle $\theta_{u^{i}-r}$.

$d_{u^{i}-r^{j}}$ is easily obtained once the user's and the resources' location are known (some coordinates systems transformations are necessary as depicted in Fig. 2). $\theta_{u^{i}-r}$ calculation, on the other hand, depends on the representation interface used by the user. For the 2D 'compass-based' representation, $\theta_{u^{i}-r} j$ is calculated as the minimum angle 
between $y^{u} w$ plane and $y^{u} y^{d}$ half-plane. In the case of the AR-based interface, this factor is obtained as the angle between $-z^{d}$ and $w$ vectors.

Priority calculation taking into account user-resource distance and angle has been modelled as a weighted sum of zero-mean Gaussian functions $\left(N_{d}\left(d_{u^{i}-r^{j}}\right)\right.$ and $N_{\theta}\left(\theta_{u^{i}-r^{j}}\right)$, respectively) as shown in (2). The relative importance of these factors can be flexibly balanced modifying $g\left(r^{j}\right)$ and $k\left(r^{j}\right)$ weights $\left(g\left(r^{j}\right)+k\left(r^{j}\right)=1\right)$.

$$
p_{s}\left(u^{i}, r^{j}\right)=g\left(r^{j}\right) N_{d}\left(d_{u^{i}-r^{j}}\right)+k\left(r^{j}\right) N_{\theta}\left(\theta_{u^{i}-r^{j}}\right)
$$

The visibility of resources is handled by including a penalization coefficient for those resources outside the area the user is located in, and also on those resources not directly visible by the user.

\section{Explicit interaction based feedback}

User-interface explicit interactions can be used in the prioritization mechanism $\left(p_{e}\left(u^{i}, r^{j}\right)\right)$. Currently, three kinds of interactions are considered:

- Resource information access event. Any resource will increase its priority if the user actively request information about it, by touching the icon associated to the resource in the user's device screen.

- Resource information access duration. The resource priority will be increased accordingly to the time the user keeps reading (if text), watching (if video), etc. the information associated to the resource.

\section{PROTOTYPE DESCRIPTION}

The above described representation strategy has been implemented in an Android-based tablet device (a Motorola Xoom powered by Honeycomb Android, v3.1) offering both $2 \mathrm{D}$ and $\mathrm{AR}$-based interfaces representing static resources, which position has been manually defined in a MySQLbased database (ResourcesKB). Whereas accurate user's location is simulated (for AR-based representation purposes), the $2 \mathrm{D}$ interface is supported by a fingerprinting method based on WiFi signal strength measurements with an average accuracy of $2-3 \mathrm{~m}$. [14]. The spatial-based prioritization method described in Section V.B has been implemented in order to change the features of the icons associated to each resource. Particularities of the implemented interfaces are detailed next.

\section{A. $2 D$ interface}

The objective of this interface is to visualize resources around the user's device in 2D format, as location and orientation biases for indoor systems are less noticeable than in 3D. Additionally, this mode may result more convenient depending on the application use.

Resources are drawn as circles with a number inside for identification purposes. A logarithmic scale (instead of a linear one) has been used for representation to facilitate interaction with the nearest points. The resulting interface (Fig. 4, up) shows a) a radar-type representation offering a $360^{\circ}$ view of the surrounding resources, b) the resources in front of the user (with an indication of the user's viewing angle; $84^{\circ}$, approximately) and c) a touch-activated legend to access resources' information. Furthermore, future works will consider points with different sizes, colour, etc. depending on their priority for the user.

Although resource representation may be handled just using local coordinates, global ones have been used (Fig. 2.a) in order to make the system seamlessly work with outdoor or indoor resources. The relative orientation of the device taking the magnetic north as reference is also calculated for representation purposes.
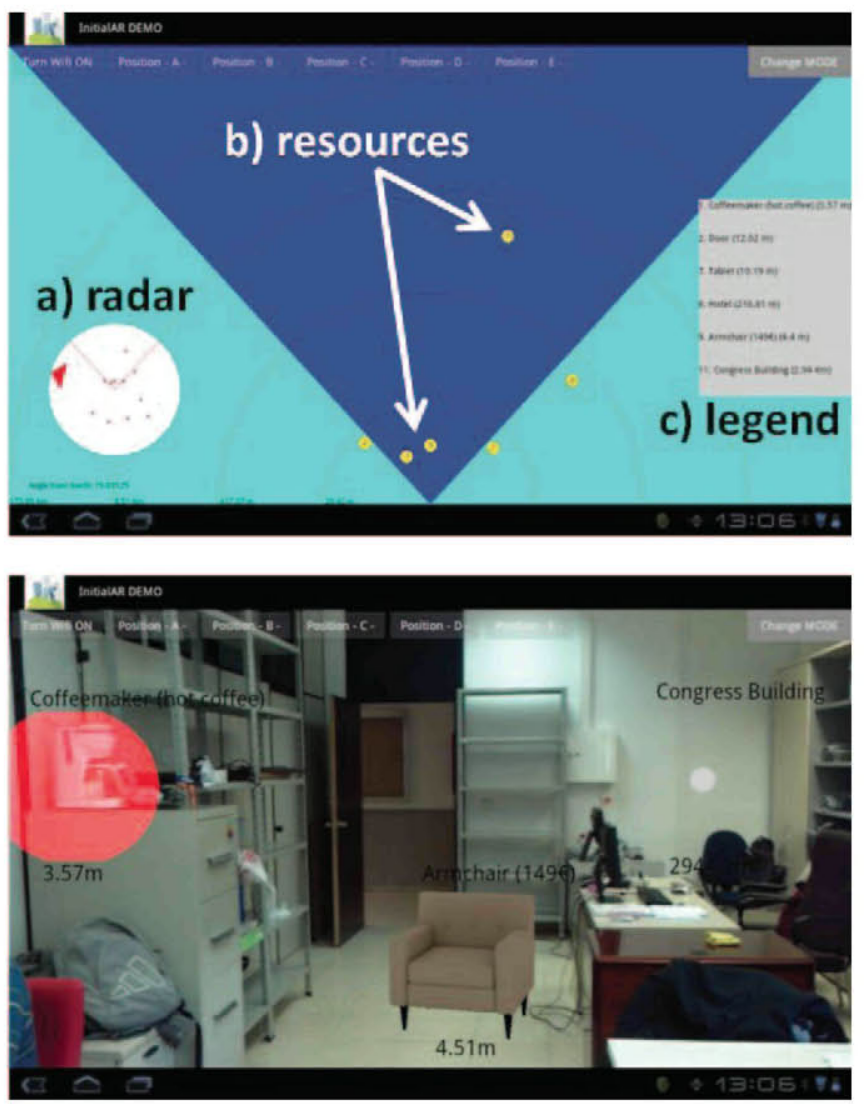

Figure 4. Resource representation interfaces; 2D (up) and AR-based (down).

\section{B. AR (3D) interface}

This mode provides a traditional AR-based view, where icons associated to real resources are superimposed in realtime on the user's device screen. 3D icons are represented using OpenGL ES ${ }^{3}$ (OpenGL for Embedded Systems). This representation relies on ${ }^{d} \mathbb{R}^{u}$, the rotation matrix relating user's and device coordinates systems (Fig. 2.b and c, respectively). ${ }^{d} \mathbb{R}^{u}$ is calculated taking into account accelerometer and magnetometer data acquired from mobile embedded sensors.

In practice, the first step for $3 \mathrm{D}$ resource representation consists of initializing the scene by loading ${ }^{d} \mathbb{R}^{u}$ into OpenGL's ModelView matrix; that configures the OpenGL

\footnotetext{
${ }^{3}$ http://www.khronos.org/opengles
} 
camera coordinates in the right direction according to device orientation. Subsequently, current user and resource's locations are used in order to correctly draw the icon associated to the resource in the device screen (repeating this process for the complete set of resources offered by the Spatial Reasoner).

AR-based resource representation will include (i) textual information about the resource (e.g., resource name or any other kind of information related to the resource) and (ii) user-resource distance (Fig. 4, down). The user may interact with the resources by touching their associated icons (internally build as an invisible sphere covering the area of the resource icon). With respect to resource prioritization, icons may vary their size, colour or opacity in order to (de)emphasize resource importance. For instance, in Fig. 4 (down) the system has assigned more priority to the resource on the left (a coffee maker), so it has displayed it on red and bigger than the resource on the right (conference building), smaller and grey-colored.

\section{CONCLUSIONS AND FUTURE WORKS}

This paper describes a mobile-based system to interact with real (and virtual) objects available in a smart space. The underlying idea is to use the augmentation capabilities of the mobile device to enable it as user-object mediator. This approach makes possible a practical and enriched development of the IoT paradigm.

In particular, the paper addresses how to build an attitude-based reasoning strategy to enhance user-object interaction and resource filtering; this strategy prioritizes the available resources depending on the spatio-temporal history of the user, his real-time location and orientation and, finally, his explicit interactions with the virtual overlay. Performance measurements of the different reasoning stages are to be accomplished in next works, together with user validation of the whole system.

The proposed reasoning method has been partially validated through a prototype that handles $2 \mathrm{D}$ and $3 \mathrm{D}$ visualization interfaces. With respect to the user experience, these interfaces have different sensitivity to location and orientation biases, being the system able to decide the visualization interface taking into account the features of the available technologies.

During the development of the prototype, we have learnt about the difficulties of implementing indoor augmented reality. This operational space offers short distance reference points to the user, being challenging to represent resources in an accurate manner. In this sense, accurate indoor position estimates should be further developed, together with smoothing techniques for resource representation stabilization.

Additionally, it is not enough with representing a $3 \mathrm{D}$ object in a given coordinates, but it is necessary to think about how it integrates with the real plane. Handling non line-of-sight resources requires a user-perception model that defines how to integrate visible and invisible resources without confusing the user. Future works consider a system to build a dynamic and easily scalable 'opacity map' providing information about resources visibility from every coordinate of the smart space.

\section{ACKNOWLEDGMENT}

This work has been supported by the Spanish Ministry of Economy and Competitiveness through the CDTI CENIT THOFU Programme and under grant TIN2011-28620-C0202 , and by the Government of Madrid under grant S2009/TIC-1485 (CONTEXTS).

\section{REFERENCES}

[1] K. Ashton, "That 'Internet of Things' Thing," In: RFID Journal, 22 July 2009.

[2] J.A. Garcia Macias, J. Alvarez-Lozano, P. Estrada-Martinez and E. Aviles-Lopez, "Browsing the Internet of Things with Sentient Visors," Computer, vol. 44, no. 5, pp. 46-52, May 2011

[3] M. Jahn, M. Jentsch, C.R. Prause, F. Pramudianto, A. Al-Akkad and R. Reiners, "The Energy Aware Smart Home," Procs. 5th Int. Conf. on Future Information Technology (FutureTech), pp.1-8, 2010.

[4] J. Carmigniani, Borko Furht, Marco Anisetti, Paolo Ceravolo, Ernesto Damiani and Misa Ivkovic, "Augmented reality technologies, systems and applications," Multimedia Tools and Applications, vol. 51, no.1, pp. 341-377, Jan. 2011.

[5] M.C. Domingo, "An overview of the Internet of Things for people with disabilities". Journal of Network and Computer Applications, vol. 35, pp.584-596, 2011.

[6] B. Hengeveld, C. Hummels, K. Overbeeke, R. Voort, H. van Balkom and J. de Moor. "Tangibles for toddlers learning language," Procs. of the 3rd International Conference on Tangible and Embedded Interaction(TEI '09). ACM, New York, NY, USA, 161-168, 2009.

[7] A. Ajanki, M. Billinghurst, et al. "An augmented reality interface to contextual information," Virtual Reality, vol 15, pp. 161-173, 2011.

[8] J. Rekimoto and Y. Ayatsuka, "CyberCode:Designing Augmented Reality Environments with Visual Tags," Proc. Designing Augmented Reality Environments, ACM Press, N.Y., pp. 1-10, 2000.

[9] P.E. Lanigan, A.M. Paulos, A.W Williams, D. Rossi and P. Narasimhan, "Trinetra: Assistive Technologies for Grocery Shopping for the Blind," Procs. 10th IEEE International Symposium on Wearable Computers, pp.147-148, 11-14 Oct. 2006.

[10] M.G.C.A. Cimino, B. Lazzerini, F. Marcelloni, G. Castellano, A.M. Fanelli and M.A Torsello, "A collaborative situation-aware scheme for mobile service recommendation," 11th Int. Conf. on Intelligent Systems Design and Applications, pp.130-135, 22-24 Nov., 2011.

11] A. Ciaramella, M.G.C.A. Cimino, B. Lazzerini, F. Marcelloni, "Using context history to personalize a resource recommender via a genetic algorithm," 10th Int. Conf. on Intelligent Systems Design and Applications, pp.965-970, Nov. 29, 2010 - Dec. 1, 2010.

[12] L. Chee Huey, P. Sebastian and M. Drieberg, "Augmented reality based indoor positioning navigation tool,", 2011 IEEE Conference on Open Systems (ICOS), pp. 256-260, 25-28 Sept. 2011.

[13] K. Jongbae and J. Heesung, "Vision-based location positioning using augmented reality for indoor navigation," IEEE Transactions on Consumer Electronics, vol. 54, no. 3, pp.954-962, August 2008.

[14] E. Metola and A.M. Bernardos, "An embedded fusion system for location management," 8 th International Conference on Mobile and Ubiquitous Systems (MobiQuitous). Copenhagen, 6-9 Dec. 2011. 